\title{
Wireless capsule retained in an ileorectal fistula in a patient with undiagnosed Crohn's disease
}
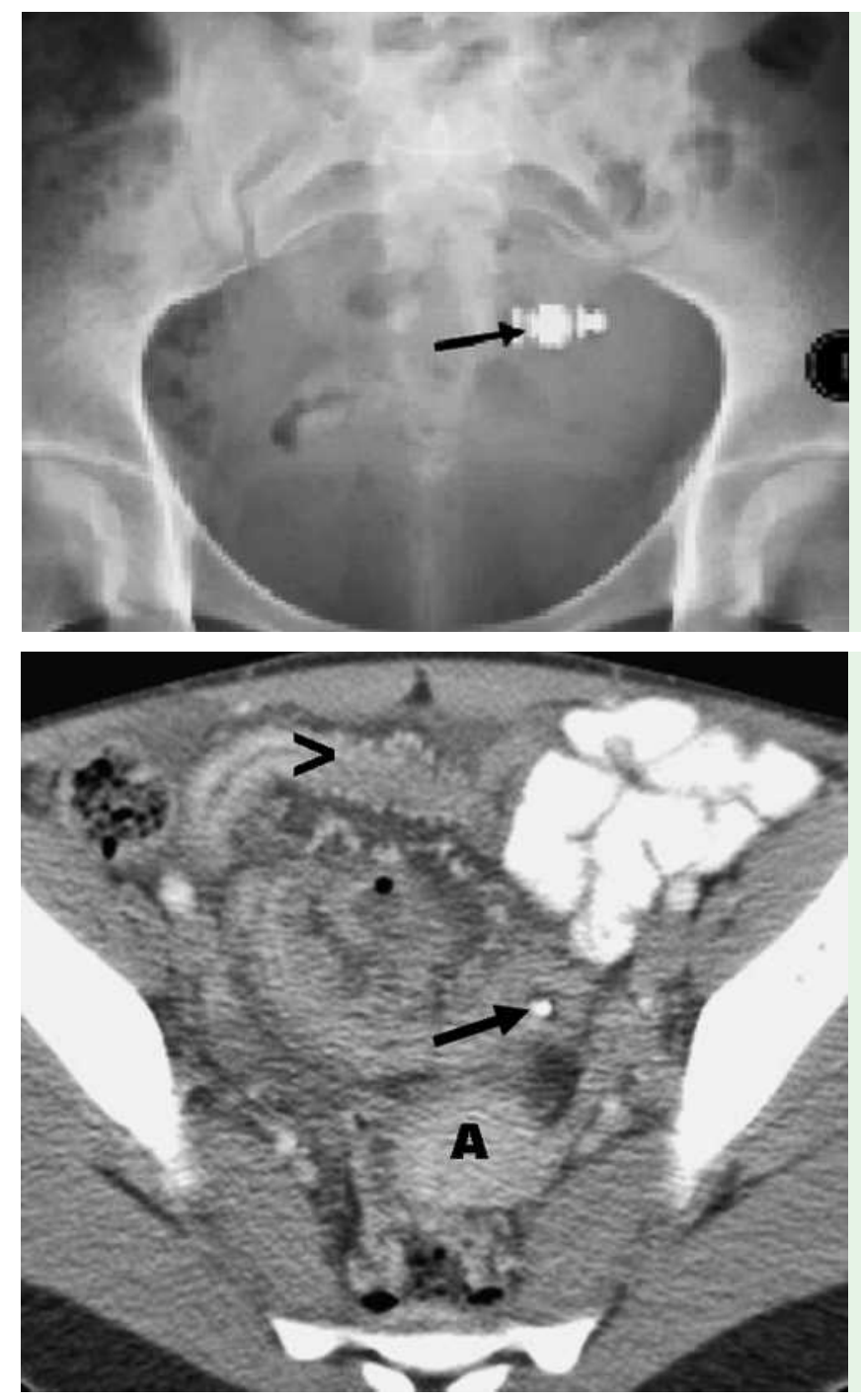

A 24-year-old woman with a presumed diagnosis of irritable bowel syndrome (with normal serum inflammatory markers, ileocolonoscopy, and small-bowel barium study) underwent video capsule endoscopy. This revealed a tight, inflamed, and ulcerated ileal stricture, which the capsule did not seem to pass through ( Video 1,2). An abdominal

\section{Video 1,2}

Wireless capsule endoscopy revealed a tight, inflamed, and ulcerated stricture, which the capsule did not appear to pass through, appearances in keeping with a diagnosis of Crohn's disease.
Fig. 1 A plain abdominal radiograph showing the capsule in the lower pelvis (arrow). There were no signs of obstruction, suggesting that the capsule had passed into the distal colon.

Fig. 2 Abdominopelvic computed tomography revealed a pelvic inflammatory mass (A) The retained capsule was identified (arrow), and there was severe ileal disease with wall thickening (arrowhead).

had passed through. An ileocecal resection with a double-barrelled stoma was performed.

Capsule retention due to small-bowel lumen strictures or stenosis has been widely reported. This complication occurs in $1.2 \%-1.6 \%$ of patients with suspected Crohn's disease and in 5\%-13\% of patients with known Crohn's disease [1,2]. This is the first report of a capsule being retained in an undiagnosed Crohn's fistula. The case also reflects how inaccurate barium studies can be in excluding significant small-bowel disease and in predicting safe passage of a capsule. Furthermore, an abdominal radiograph can be misleading in localizing the position of a capsule (it appeared to be in the distal colon according to the radiographic evidence in this case).

Capsule retention in an unrecognized Crohn's fistula is therefore a potential complication of video capsule endoscopy, and one that necessitates urgent surgical treatment. An abdominal radiograph can be misleading in determining the location of a retained capsule and a computed tomographic scan should be considered for all patients with suspicious symptoms.

\section{Endoscopy_UCTN_Code_CPL_1AI_2AB}

\section{C. Sulz, S. H. Anderson}

Department of Gastroenterology and Hepatology, St. Thomas' Hospital, Guy's and St. Thomas' NHS Foundation Trust, London, United Kingdom

\section{References}

1 Kornbluth A, Colombel JF. ICCE consensus for inflammatory bowel disease. Endoscopy 2005; 37: 1051

2 Cheifetz AS, Kornbluth AA, Legnani $P$ et al. The risk of retention of the capsule endoscope in patients with known or suspected Crohn's disease. Am J Gastroenterol 2006; 101: $2218-2222$

radiograph 18 hours later showed the capsule in the lower pelvis, and there were no signs of obstruction, suggesting that the capsule had passed into the distal colon ( Fig. 1).

The patient developed worsening abdominal pain and abdominopelvic computed tomography revealed severe ileal disease with an inflammatory mass and the retained capsule ( $\bullet$ Fig. 2). At laparotomy a large, indurated ileocecal mass with fistulation into the rectum was found, in keeping with Crohn's disease. The capsule was located in the fistula, not within the intestinal lumen. There was an ileal stricture $(10 \mathrm{~cm}$ in length, $30 \mathrm{~cm}$ proximal to the ileocecal valve) which the capsule
Bibliography

DOI 10.1055/s-2007-967057

Endoscopy 2008; 40: E5

(c) Georg Thieme Verlag KG Stuttgart · New York . ISSN 0013-726X

\section{Corresponding author}

\section{S. H. Anderson, MD}

Department of Gastroenterology and Hepatology St. Thomas' Hospital

London SE1 7EH

United Kingdom

Fax: +44-207-188-2484

simon.anderson@gstt.nhs.uk 